\title{
A NOVEl APPROACH FOR OPTIMIZING ENERGY AND BANDWIDTH IsSUES IN MANET USING DSR PROTOCOL
}

\author{
Ajay Lala, Anand Bhaskar, Prasun Chakrabarti and JitendraKaushal Srivastava \\ Department of Computer Science \& Engineering, Sir PadampatSinghania University, \\ Udaipur, Rajasthan, India
}

\section{ABSTRACT}

An ad-hoc network is a cluster of wireless mobiles nodes which are self-making, self-arranging and selfregulating. Numerous energy conserving routing conventions have been proposed for Mobile Ad Hoc Network (MANET). Those conventions demonstrate that energy utilization in MANET can be reduced by modifying the transmit energy to a base level. To decide the base transmit power is a testing issue. This paper proposes a novel approach for optimizing Energy Issues in MANET using DSR Protocol convention which guarantees high reliability of the system. The Dynamic Source Routing (DSR) convention has been adjusted with a specific end goal to execute IE-DSR convention. Existing control messages of DSR convention have been adjusted and utilized as a part of IE-DSR convention. The proposed method introduces a systematic model to demonstrate that an impressive part of energy can be spared by utilizing $I E-D S R$ convention. This calculation lessens the flooding of RREQ (route request) packets in the network which brings about the reduced energy utilization. The proposed calculation precludes the superfluous flooding of RREQ packets.

Based on the experimental work, the proposed method outperforms in reducing energy consumption by a rationale factor. Further, the simulation results depict that if the initial energy of node is kept relatively small then our proposed approach consumes approx. $70 \%$ less energy than DSR, which is quite significant while comparing with the existing energy optimizing methods and it has been analyzed that an $15.36 \%$ of more data packet has been delivered to the appropriate destination. The application of the proposed method ensures larger network life, high reliability and lower cost estimation.

\section{KEYWORDS}

Ad-hoc Networks, IE-DSR, Flooding, RREQ, Routing Protocols;

\section{INTRODUCTION}

In Ad-hoc networks are wireless networks that have no fixed infrastructure. Such kind of networks is created dynamically and they provide special challenges beyond those the network. The structure of wireless ad-hoc network was initially designed to be a flat type, which means that the ad- hoc network is assumed to be homogeneous. Based on the flat structure, the routing work can be simply done by flooding. However, when the number of nodes in the network grows to hundred or even thousand, the mass control message will even block the data transmission. It has been proved that even under the optimal circumstances, the throughput for each node declines rapidly towards zero while the number of nodes is increased. Because ad-hoc networks do not rely on existing infrastructure and are self-organizing, they can be rapidly deployed to provide robust communication in variety of hostile environments [1]. A wireless ad-hoc network comprises of different portable self - sorted out and battery operated wireless devices having diverse battery limit and power scattering. Consequently battery lifetime turns into a key component that influences the exhibitions of mobile ad-hoc network. Different route discovery

DOI: $10.5121 / \mathrm{ijcnc} .2017 .9502$ 
components utilizing DSR for MANET have been given in writing. In [2] the author proposed a mechanism for highway environment which is based on the feature of vehicle movement. The work done in [3] proposes a route determination instrument and versatility expectation for making DSR a vitality effective routing convention, in this creator explored the effect of portability on the execution of an on-request control mindful directing convention. In [4] creator proposed a calculation which chooses a route having the best accessible transfer speed. Flooding administration in ad-hoc network by controlling the portability and transmission power is appeared in [5]. Different ad-hoc routing protocols and their examination is appeared in [6]. In [7] author proposed the mechanism for reducing the route request flooding attack. The design and performance evaluation of a secure on-demand ad-hoc network routing protocol has been shown in [8]. It prevents many types of denial-of-service attacks. In [9] creator proposed a system that can diminish the routing overhead by using earlier directing histories.

In present work, we have proposed a proficient and straightforward method which lessens the quantity of RREQ packets overwhelmed with a specific end goal to improve the execution of an Ad-hoc network. This algorithm considers the change in route revelation system at intermediate nodes. The change procedure, incorporates the checking of parameters like mobility, residual battery and received signal strength of node itself by the defined condition. In this procedure those nodes are disposed of which don't have the characterized conditions. In this manner, those nodes won't further forward route request packets. Hence congestion the network will reduce.

An energy efficient routing protocol decreases the power consumption of the nodes by routing data on paths that consume the least amount of energy. There are some special mechanisms to achieve this goal. Due to changing technologies and new researchers for finding the optimal DSR protocol solution, many significant enhancement and modification have been done to make DSR an Energy efficient routing protocol. This section discusses some important routing protocols which are made by doing modification in traditional.

\section{RELATED WORK}

The work in [10] proposes another modified DSR protocol which is aimed to prolong the network life time by using basic two approaches of power consumption, one is transmission power control approach and the second one is load balancing approach. The work in [11] proposes MDSR protocol aimed to reduce overhead by reducing the number of routing reply packets and a fixed header size for the data packets and acts itself as one of the energy efficient routing protocol. In [12] creator has proposed some new structure for the control packets to change the behaviour of the nodes implements a new Energy table and creates a new algorithm for route cache and route selection. The simulation result shows that E2DSR has better performance than DSR. And it has left many doors opened for future studies and research works because all these metrics described above, have not been implemented yet for large scenario.

Above methodologies suggest that energy aware routing protocols save energy. In any case, that convention brings about extra control overhead this can expand superfluous transmission capacity of the network. Extra equipment or programming is required in a few conventions, which may not be plausible for the portable nodes since versatile nodes ordinarily have low preparing forced and constrained equipment assets. In this paper, we propose a base energy directing convention called Improvised Energy Dynamic Source Routing (IEDSR) convention. Our approach is to keep away from extra control message. Existing control packets of DSR convention have been utilized as a part of request to execute the IEDSR convention. The IEDSR convention works in two stages' route disclosure and connection by connection control change. Two power levels have been utilized amid the route revelation period of the convention. At initial, a source node starts the route revelation to discover a route to its goal by communicating a request packet at low power 
International Journal of Computer Networks \& Communications (IJCNC) Vol.9, No.5, September 2017

level. On the off chance that the source node finds a way utilizing that power level, it set up the association utilizing that power level. On the off chance that a source node can't find a route to its goal utilizing that low power level in the wake of starting route disclosure for a specific number of times, it accepts that the goal is not reachable at that power level. At that point it increases the power level to a higher esteem and starts the route disclosure again at that power level.

Recently an Intelligent Energy-aware Efficient Routing protocol for MANET (IE2R) is proposed [16]. In this research the author proposed technique based on entropy and Preference Ranking Organization Method for Enrichment of Evaluations-II. They have simulated the proposed method in NS2 and claims the results obtained outperforms existing protocols in terms of several network metrics.

The remaining part of the paper is arranged as follow: In section 3, we will clarify requirement for lessening the route requests for in specially appointed network. In section 4 , we will clarify the DSR convention in brief. In section 5, we will display the IEDSR convention in detail. In section 6 we will demonstrate some simulation results and section 7 will conclude our works.

\section{Requirement For Reducing The Route Request In Ad-Hoc NETWORK}

The dynamic source routing protocol (DSR) is a reactive MANET routing protocol. When imparting a goal node which is absent in the route cache of the source node, it will buffer the information packets and communicate a course ask for (RREQ) into the system. The other middle DSR nodes communicate the RREQ packet to the expected goal node. The destination node then will send a Route Reply (RREP) on the reverse route back to the source node.

In the event that no RREP is received after a settled number of endeavours and within the inside net_traversal_time, the information packets from the buffer will be dropped. On the off chance that more information packets are gotten, another course revelation process will be started. Expanding the number of route requests will soak the system which makes it incapable for further transmission of information packets [6].

A reactive routing protocol, by and large, requires less routing overhead than proactive routing protocols since they take a shot at request. Proactive routing protocols are no influenced by expansive number of flooding of course demand in light of the fact that if the goals are not discovered, information packets are dropped at the source or any middle of the road nodes. However, it can truly corrupt the execution of reactive routing protocols and influence a node in the following ways:

- During the procedure of course revelation the information packets are cradled in the memory. What's more, if the extensive quantities of information packets beginning from application layer are really inaccessible, honest to goodness information packets in the cushion memory might be supplanted by these inaccessible information packets.

- The expanded number of RREQ packets in the system brings about more impacts and subsequently, blockage and additionally postpone increment for the information packets in the system.

Power and data transfer capacity administration is imperative in MANET and transmission of expansive number of RREQ can expand the power and transfer speed utilization. 


\section{BASIC DSR OPERATION}

The Dynamic Source Routing protocol (DSR) is a routing protocol for a multihop mobile ad hoc network. This protocol does not require any pre-installed infrastructure and hence self-configuring and self-organizing. The protocol is discussed below:

\subsection{ROUTE DISCOVERY}

This mechanism is initiated whenever a node wishes to transmit or communicate a destination node which isn't in its transmission range. The route Discovery process is initiated to find a route to that node which is outside the transmission range of the sender. First the route cache is searched; if the route is not in the cache then following steps are performed to find the route:

- It generates a route request packet, which contains the address of its own and the destination node; then this packet is broadcast to all its neighbours using flooding.

- When this request is received by neighbours, they consult with their own cache to find an eventual route to this destination to be sent back to the sender; otherwise it rebroadcast the same route request to all of its neighbours after including its address to the header of the route request and learns from this request information to be added to its cache. If the node already has information about this route request it ignores the newly received request by verifying its sequence number because sequence number is unique for each route request.

The similar mechanism is used by each neighbouring node until the route request arrives to destination which includes its address at the end of the header and sends a route reply.

\subsection{ROUTE REPLY}

This process is executed by the node after receiving the route request packet destined for him, and performs the below mentioned actions:

- This new route is added to its cache for future use.

- Its address is added at the end of the path contained in the DSR packets header.

- Replies in uni-casting manner to this request the path contained in the header

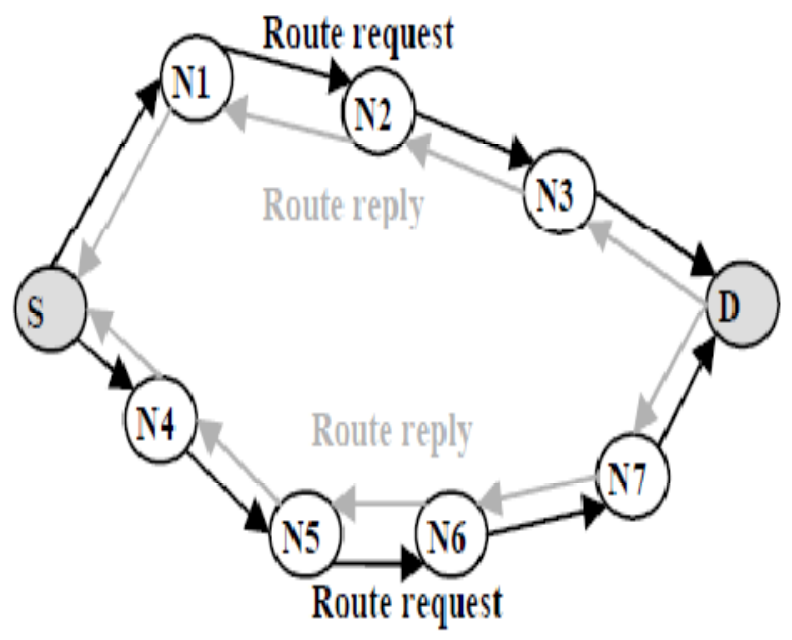

Figure 1. Route Discovery Process 


\subsection{Route MaintenanCE}

When forwarding each intermediated node is responsible for confirming that packet must be correctly received by the next node but due to some wireless medium constraints, the node doesn't receive the acknowledgement of reception and hence resends packet until a predefined attempt is reached. As the no of attempts is reached the node consider this link as broken and generates a route error packet to inform source node and all intermediate nodes about the link failure. All the intermediate nodes remove its entries from the table regarding that route and forward this error packet to others until arrives at its destination which chooses to launch a new route request or to identify a new route in route cache of its own.

\subsection{Route Cache}

In DSR, the route cache is used to manage regularly used routes to avoid new route discovery mechanism which require a lot of network resources. The new identified route is stored in the route cache of the respective node for future use; the newly routes are also learned by a node through route request and the route error packets.

\subsection{Metric}

The No of hops is the metric used to choose the best route from source node to destination node. Since an existing route has minimum no of hops it may exist a route with the minimum of hops however it contains some less powered or unstable nodes those may generate link failure and therefore route errors.

\section{Proposed IEDSR Algorithm}

The flow chart of proposed algorithm viz. IEDSR is shown in figure 2.

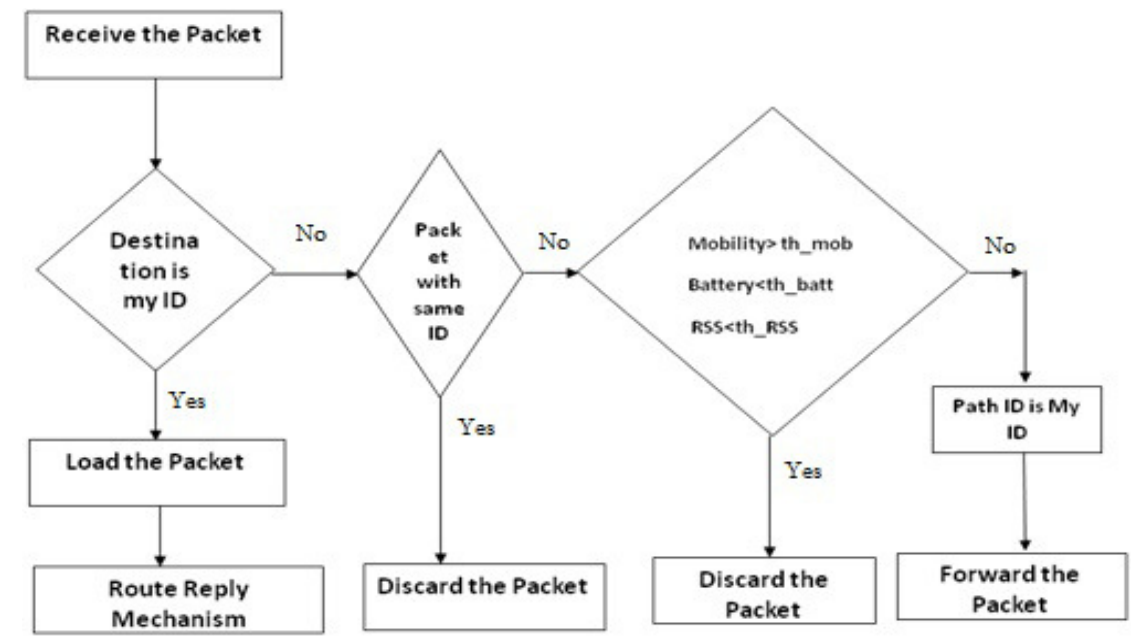

Figure 2.Flow Chart of Route Discovery Mechanism in IEDSR

It shows the mechanism of forwarding the route request packets by the intermediate nodes. For proposed IEDSR the modifications made in route discovery process only, which is as follows: 
Step 1. When RREQ flooded, it reaches to the nodes which are in the transmission range of source node. When a node receives a RREQ packet, following conditions/possibilities will be checked:

If it is the destination node, it sends a route reply to the sender.

Else

Step 2. If this node receiving the route request has recently seen another route request message from this initiator bearing the same request identification and target address, or if this node's own address is already listed in the route record in the route request, this node discard the request.

Else:

Step 3. If \{value of the speed of the node is greater than $10.41 \mathrm{~m} / \mathrm{sec}$ OR value of the residual battery is less than the threshold value of residual battery i.e. $=142$ Joule OR value of the received signal strength is less than the threshold received signal strength i.e. $=-85$ $\mathrm{dBm}\}$

Step 4. Discard the request.

Else

Step 5. The node adds its own address to the request packet and forwards it.

\section{Simulation RESUlt}

A network simulator software that predicts the behaviour of a computer network. In simulators, the computer network is typically modelled with devices, links, applications etc. and the performance is analyzed. Typically, users can then customize the simulator to fulfil their specific analysis needs [13].

A large portion of the business simulators is GUI driven, while some network simulators are CLI driven. The network model / setup depict the condition of the network (hubs, switches, switches, and links) and the events (information transmissions, packet errors and so forth). An important output of simulations is the trace files. Trace files log every packet, every event that occurred in the simulation and is used for analysis. Network simulators can also provide other tools to facilitate visual analysis of trends and potential trouble spots. Most network simulators use discrete event simulation, in which a list of pending "events" is stored, and those events are processed in order, with some events triggering future events such as the event of the arrival of a packet at one node triggering the event of the arrival of that packet at a downstream node. Simulation of networks is a very complex task. For example, if congestion is high, then estimation of the average occupancy is challenging because of high variance. To estimate the likelihood of a buffer overflow in a network, the time required for an accurate reply can be extremely large. Specialized techniques such as "control variants" and "importance sampling" have been developed to speed simulation $[14,15]$.

Simulation Procedure:

- Simulation Object Creation

- Tracing (Simulation Object - NAM)

- Topology Formation

- Communication Agent

- Traffic Model

- Error Model

- Algorithm Designing

- Run the Object

These are all steps for completing the simulation modelling process for entire common research work. 


\subsection{ENERgY CONSUMPTION MODEL}

A generic expression to compute the energy required to transmit packet $\mathrm{p}$ is:

$$
\mathrm{E}(\mathrm{p})=\mathrm{i} * \mathrm{v} * \text { tp Joules, }
$$

where: $\mathrm{i}$ is the present utilization, $\mathrm{v}$ is the voltage utilized, and to the time required to transmit the packet. We assume that every cell phone is furnished with IEEE $802.11 \mathrm{~b}$ system interface cards (NICs). The energy utilization esteems were received by contrasting business items and the trial information announced in [11].

The qualities utilized for the voltage and the packet transmission time was: $\mathrm{v}=5 \mathrm{~V}$ and up= $(\mathrm{ph} / 2 * 106+\mathrm{pd} / 11 * 106) \mathrm{sec}$, where $\mathrm{ph}$ and PD are the packet header and payload measured in bits, separately. We computed the energy required to transmit and get a packet $p$ by utilizing:

$$
\operatorname{Etx}(p)=280 m A * v * \operatorname{tp} \text { and } \operatorname{Erx}(p)=240 m A * v * \operatorname{tp}
$$

Besides, we represent energy spent by nodes catching packets. As appeared in [11], we accept the energy utilization brought on by catching information transmission is the same as that devoured by really getting the packet. With the end goal of assessing the impact of catching, we changed the energy model to account not for the energy use because of transmission and gathering additionally the battery cost to be consumed by catching the remote channel. Therefore, the aggregate sum of energy, $\mathrm{E}(\mathrm{Ni})$, consumed at a node $\mathrm{Ni}$ is determined as:

$$
\mathrm{E}(\mathrm{ni})=\operatorname{Etx}(\mathrm{ni})+\operatorname{Erx}(\mathrm{ni})+(\mathrm{N}-1) * \operatorname{Eo}(\text { ni })(1)
$$

Where Etx, Erx, and Eo mean the measure of energy use by transmission, gathering, and catching of a packet, individually. $\mathrm{N}$ speaks to the normal number of neighbouring nodes influenced by a transmission from node Ni. Eq.(1) infers that when the system is more thicker, the packet catching causes more energy utilization.

\subsection{Simulation ANALYSIS}

We test the execution of IEDSR convention by utilizing NS2.Constant Bit (CBR) traffic utilized for creating information packet rate of 1 packet/sec. Each CBR traffic starts at random time during the simulation period. We analyze the performance of IE DSR protocol with two different types of energy constraints- (l) mobile nodes are assigned a large amount of energy and the simulation is run for a limited period of time, and (2) mobile nodes are assigned a very limited initial energy and the simulation is run for a long period of time.

\section{Case I}

Initial energy supply to each node 300 joules, simulation time 250 seconds. Table 1.Threshold Values of Various Parameters for IEDSR 


\begin{tabular}{|l|l|}
\hline Parameter & Routing protocol-DSR \\
\hline $\begin{array}{l}\text { Threshold value of residual } \\
\text { battery }\end{array}$ & 142 Joule \\
\hline $\begin{array}{l}\text { Threshold value of speed of node } \\
(\mathrm{m} / \mathrm{sec})\end{array}$ & $10.41 \mathrm{~m} / \mathrm{sec}$ \\
\hline Threshold value of RSS & $-85 \mathrm{dBm}$ \\
\hline
\end{tabular}

The energy consumption with DSR and IDSR (with threshold 142 joule) is as follows:

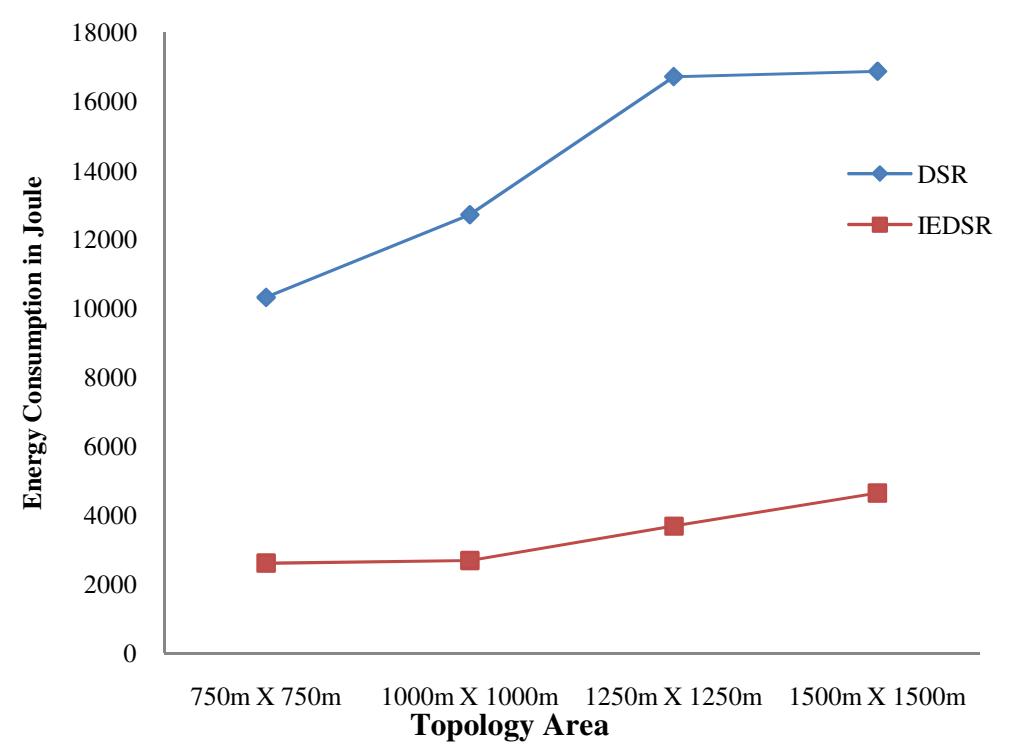

Figure 3.Energy Consumption by 100 Mobile Nodes in DSR and IEDSR

From figure. 4 we can conclude that Energy consumption is increased by $74.7 \%$ from $750 \mathrm{~m} \mathrm{x}$ $750 \mathrm{~m}$ to $1000 \mathrm{~m} \times 1000 \mathrm{~m}$ topology area, which is further increased up to $78.8 \%$ from $1000 \mathrm{~m} \mathrm{X}$ $1000 \mathrm{~m}$ to $1250 \mathrm{~m} X 1250 \mathrm{~m}$ topology area respectively. The energy consumption per data packet increases in DSR protocol as the network size increases. The reason is that the data packets are travelling more hops in this protocol when we place the same number of nodes in larger network size. 
International Journal of Computer Networks \& Communications (IJCNC) Vol.9, No.5, September 2017

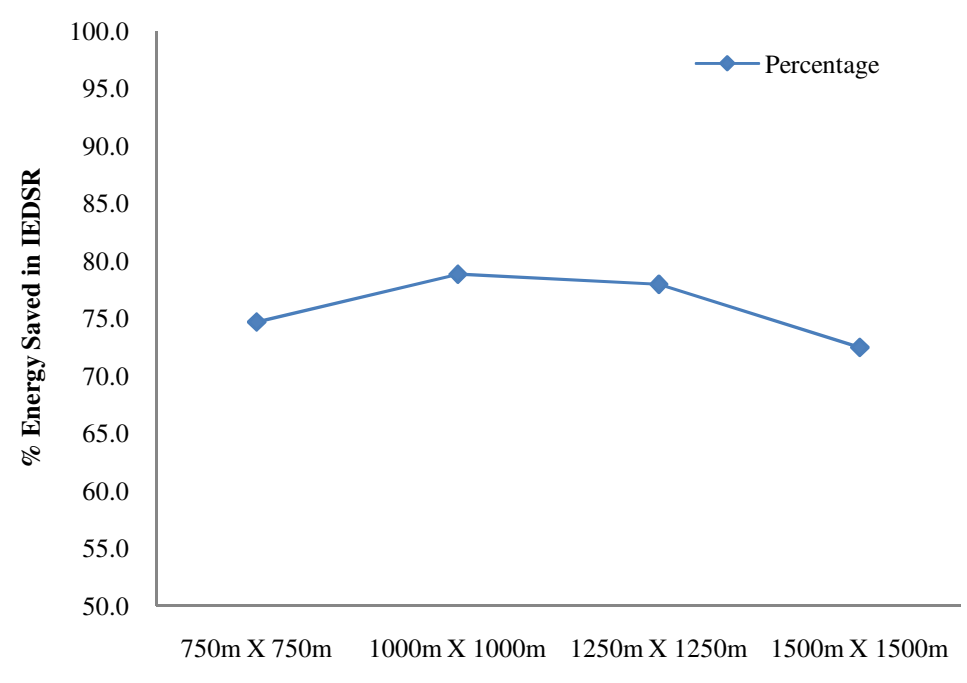

Topology Area

Figure 4.Percentage Energy Saving in IEDSR

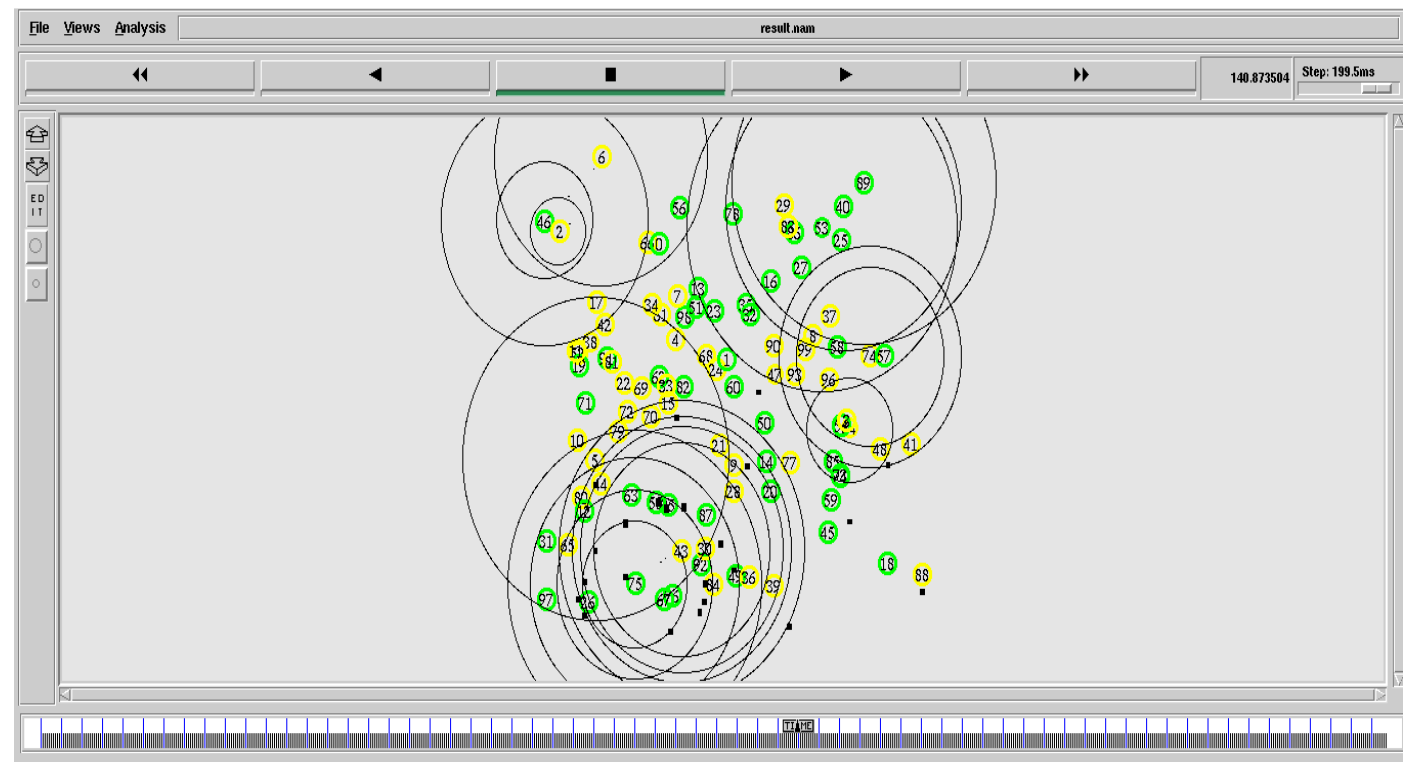

Figure 5. Simulation Scenario of Energy Consumption at 140.84 seconds

\section{Case II}

Initial energy supply to each node 400 joules, simulation time 250 seconds. The energy consumption with DSR and IDSR (with threshold 142 joule) is as follows: 
International Journal of Computer Networks \& Communications (IJCNC) Vol.9, No.5, September 2017

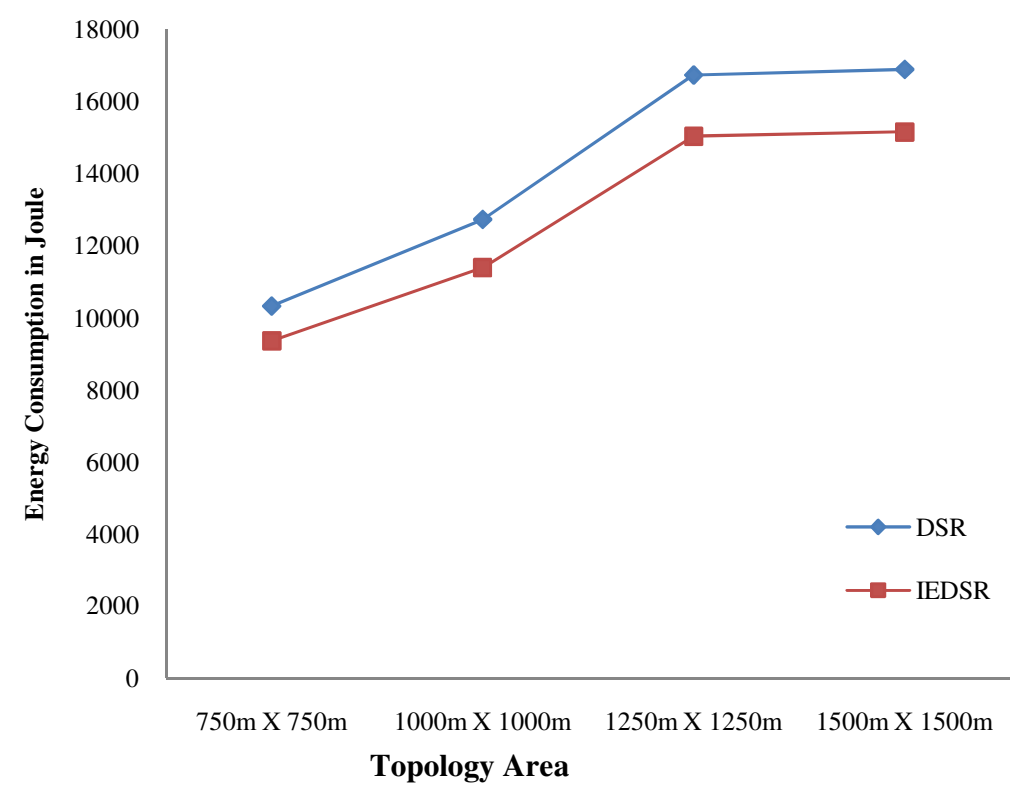

Figure 6.Energy Consumption by 100 Mobile Nodes in DSR and IEDSR

Figure 7 concludes that Energy consumption is increased by $9.4 \%$ to $10.5 \%$ from $750 \mathrm{~m} \times 750 \mathrm{~m}$ to $1000 \mathrm{~m}$ X $1000 \mathrm{~m}$ topology area, which is further decreased up to $10.2 \%$ from $1000 \mathrm{~m}$ X $1000 \mathrm{~m}$ to $1250 \mathrm{~m}$ X $1250 \mathrm{~m}$ topology area respectively. The energy consumption per data packet increases in DSR protocol as the network size increases. The reason is that the data packets are travelling more hops in this protocol when we place the same number of nodes in larger network size.

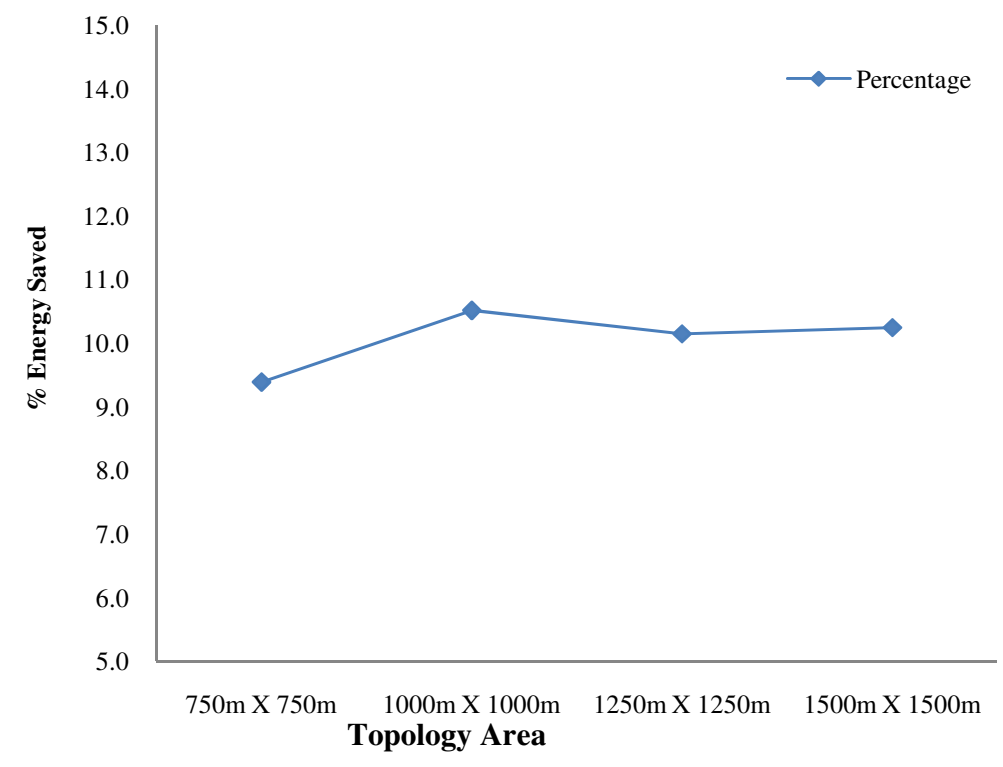

Figure 7.Percentage Energy Saving in IEDSR 


\section{Case III}

In this case, we randomly place 100 nodes in an area of $750 \mathrm{~m}$ by $750 \mathrm{~m}$. The other parameters are same as in the previous simulation. Only the network area is increased in this case to ensure that more number of nodes participating in the network operation. We assign a limited initial energy of 300 Joules to each mobile node and run the simulations for 250 seconds. The packet transfer stops at certain point during the simulation when all connections are disconnected due to node failures. This gives us an estimate about how many data packets can be sent successfully to the destination by a particular network when the mobile nodes have very limited batteries. The simulations results are shown in figure 8. It can be concluded from that figure that when the network size is the smallest which is $750 \mathrm{~m}$ by $750 \mathrm{~m}$, almost $21.62 \%$ more data packet was delivered to the destination by using the IEDSR protocol. We then increase the network area to $1000 \mathrm{~m}$ by $1000 \mathrm{~m}, 1250 \mathrm{~m}$ by $1250 \mathrm{~m}$, and $1500 \mathrm{~m}$ by $1500 \mathrm{~m}$. It has been analyzed that as the size of network grows, both approach has similar behaviour in terms of packet delivery. Although the packet delivery ratio of both the algorithm DSR \& IE-DSR exchanges similarity in multiple instances but the proposed IE-DSR has higher packet delivery ratio with compare to the existing approach. The reason of this increase in packet delivery ratio reduction is congestion, bandwidth consumption and delay.

Table 2.Simulation Parameters Used For DSR \& IE-DSR

\begin{tabular}{|c|c|c|c|c|c|c|c|c|}
\hline \multirow[b]{2}{*}{ Parameters } & \multicolumn{2}{|c|}{$750 \mathrm{mX750m}$} & \multicolumn{2}{|c|}{$1000 \mathrm{mX1000m}$} & \multicolumn{2}{|c|}{$1250 \mathrm{mX1250m}$} & \multicolumn{2}{|c|}{$1500 \mathrm{mX1500m}$} \\
\hline & DSR & $\begin{array}{l}\text { IE } \\
\text { DSR }\end{array}$ & DSR & $\begin{array}{l}\text { IE } \\
\text { DSR }\end{array}$ & DSR & $\begin{array}{l}\text { IE } \\
\text { DSR }\end{array}$ & DSR & $\begin{array}{l}\text { IE } \\
\text { DSR }\end{array}$ \\
\hline $\begin{array}{l}\text { Send } \\
\text { Packets }\end{array}$ & $\begin{array}{l}1199 \\
3\end{array}$ & 4595 & 12001 & 4615 & 11987 & 4542 & 11994 & 4637 \\
\hline $\begin{array}{l}\text { Received } \\
\text { Packets }\end{array}$ & 1713 & 1650 & 2017 & 1621 & 1070 & 731 & 2179 & 1508 \\
\hline $\begin{array}{l}\text { Packet } \\
\text { Delivery } \\
\text { Ratio \% }\end{array}$ & 14.28 & 35.09 & 16.8 & 35.12 & 8.92 & 16.09 & 18.16 & 32.52 \\
\hline $\begin{array}{l}\text { Total } \\
\text { Dropped } \\
\text { Packets }\end{array}$ & $\begin{array}{l}3359 \\
91\end{array}$ & 115310 & 309699 & 110759 & 361243 & 153219 & 292907 & $\begin{array}{l}1021 \\
30\end{array}$ \\
\hline $\begin{array}{l}\text { Normalized } \\
\text { Routing } \\
\text { Load }\end{array}$ & $\begin{array}{l}1419 \\
457\end{array}$ & 496283 & 1381101 & 507208 & 1681548 & 754149 & 1432956 & $\begin{array}{l}5110 \\
19\end{array}$ \\
\hline $\begin{array}{l}\text { Average } \\
\text { End to End } \\
\text { Delay in ms }\end{array}$ & $\begin{array}{l}3411 . \\
0\end{array}$ & 3191.2 & 6433.56 & 3900.8 & 7426.3 & 6340.3 & 4005.3 & $\begin{array}{l}3775 . \\
1\end{array}$ \\
\hline
\end{tabular}




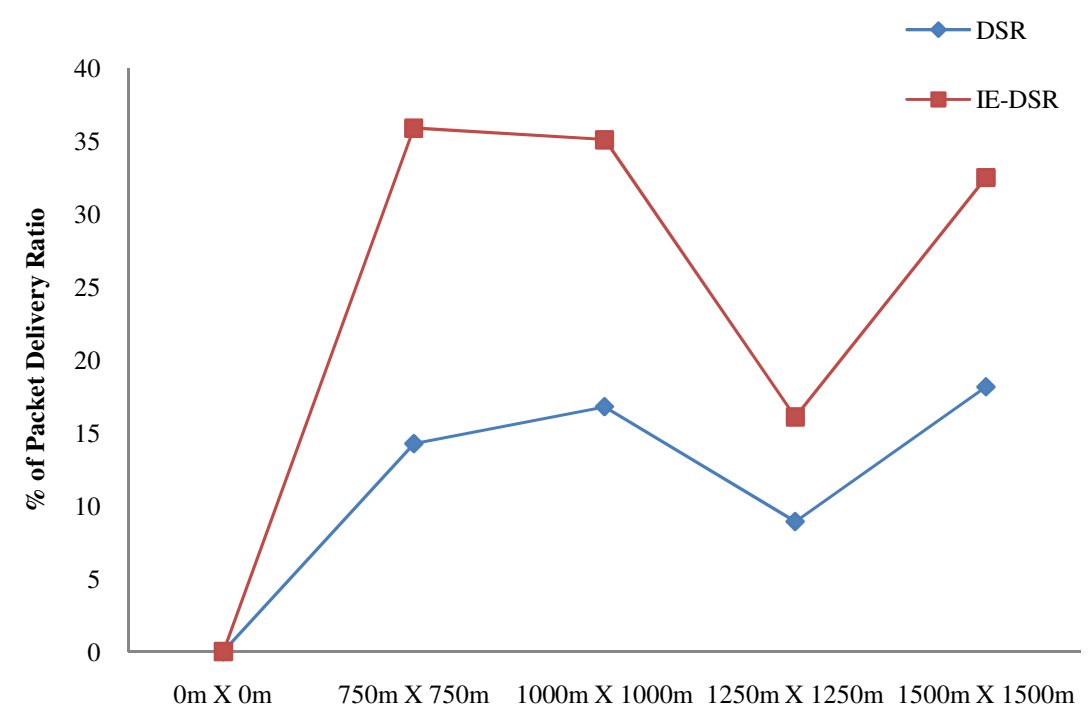

Topology Area

Figure 8.PDR in \% with 300 Joules DSR and IEDSR

\section{Conclusions}

A need for controlling the flooding of RREQ is a very important task in MANET because it consumes more power of the network. It also causes high congestion and delay in the network. The IEDSR will provide a better solution to overcome it. In IEDSR route discovery mechanism reduces energy consumption by not taking into account those nodes whose battery is lesser, mobility and distance is greater than the defined threshold level. If following assumptions are not considered the chances of network failure increases. Because high speed nodes will come into a network for short time period and the node having lesser battery charge will not remain on a network for a long duration. Based on our experimental work, the proposed method outperforms in reducing energy consumption by a rational factor. Further, the simulation results depict that if the initial energy of the node is kept relatively small then our proposed approach consumes approx. $70 \%$ less energy than DSR, which is quite significant while comparing with the existing energy optimizing methods and it has been analyzed that a $15.36 \%$ of more data the packet has been delivered to the appropriate destination. In all, IEDSR outperforms the existing DSR. This protocol can be useful at the place where the node density is very high and delay is to be minimized. Furthermore, it has left numerous doors opened for future reviews and research works since all these metrics described above, have not been implemented yet for a large scenario. So we may get many interesting outcomes while carrying out any experiment with a larger scenario with all these performance matrices. 


\section{REFERENCES}

[1] Cheng ,R.H , Wu,T.K , Yu,w.c. , "A highly topology adaptable ad hoc routing protocol with complementary preemptive link breaking avoidance and path shortening mechanisms", Wireless Network, DOl 10. I 007/s11276-009-0203-0, pp. 1218-1311, 2010.

[2] Gang, Z., Han, T., Wenwei S., Chunfeng, L., Yantai, S., "A Hybrid DTN-DSR Routing protocol Based on Clustering", 8th International Conference on wireless communication Networking and mobile Computing (WiCOM), doi:10.1109/WiCOM.2012.6478291, 2012.

[3] Luo, Y., Wang, J., Chen, S., "An energy efficient DSR routing protocol based on mobility", World of wireless, International Symposium on Mobile and Multimedia Networks, doi:10.1109/WOWMOM.2006.25 publication, 2006.

[4] Kadri, B., Moussaoui, D., Feham, M., "A cross-layer design for QoS impletion in MANETs applied to DSR", 3rd International Conference on Infonnation and Communication Technologies doi:10.1109/ICTTA.2008.4530206.

[5] Smys, s., Bala, G. J., Jennifer, S., "Mobility management in wireless networks using power aware routing", International Conference on Intelligent and Advanced System (ICIAS), doi: 10.1109/ICIAS.2010.5716234.

[6] Jacob, J., Seethalakshrni, V., Performance Evaluation of Various Routing Protocols in MANET, An International Journal of Engineering Sciences, 5, pp.208-220, 2011.

[7] ZhiAng EU, Winston Khoon Guan seah, "Mitigating Route request Flooding Attacks in Mobile Adhoc Networks", International Conference on Information Networking doi: 10.1007/1191956833, pp. 327-336, 2006.

[8] Hu, Y.C., Perrig, A., Johnson, D. B., Ariadne, "A Secure OnDemand Routing Protocol for Ad-hoc Networks", Springer Science, Wireless Networks, vol. 11, pp. 21-38, 2005.

[9] Castaneda, R. , Das, S. R., Marina, M. K., "Query Localization Technique for On-demand Routing Protocols in Ad-hoc Networks", Kluwer Academic Publishers, Wireless Networks vol. 8, pp. 137$151,2002$.

[10] Tarique M., Tepe K. and Naserian M., "Energy Saving Dynamic Source Routing for Ad-hoc Wireless Networks “, In Proc of IEEE -WIOPT, April 3-7 (2005), p.305-310.

[11] Timlarasi M., Chandramithi S. and Palanivelu T. G., Overhead Reduction and Energy Management in DSR for MANET, In Proc. of IEEE-COMSWARE, January 6-10 (2008), p. 762-766.

[12] Vahid N. T., Hugo M., Jonathan R. and Hugo A. An Energy Efficient Flat Routing for Mobile Ad-hoc Networks, In Proc. of IEEE-ICCCN, August 2-5 (2010),p.1-6.

[13] Palak, Nasib Singh Gill, “A POWER SAVING INTELLIGENT DYNAMIC SOURCE ROUTING (IDSR) IN MANET", International Journal of Advanced Research in Computer and Communication Engineering, Vol. 3, Issue 7, July 2014.

[14] SimarPreet Singh, Barinderpal Singh, "ROUTING ALGORITHM IN MANET", International Journal of Engineering and Innovative Technology (IJEIT), Vol. 3, Issue 9, March 2014.

[15] Nancy A., "Enhanced Dynamic Source Routing Algorithm for Energy Efficiency in MANET”, SSRG International Journal of Computer Science \& Engineering (SSRG-IJCSE), 2(8), 7 (2015).

[16] Das, Santosh Kumar, and Sachin Tripathi. "Intelligent energy-aware efficient routing for MANET." Wireless Networks (2016): 1-21. 